\title{
Study on Real-Time Measurement and Control Tap of Electric Power
}

\author{
Masashi Ohchi $^{1 *}$ and Tomonobu Tanaka ${ }^{2}$ \\ ${ }^{1}$ Department of Electrical and Electronics Engineering Chiba Institute of Technology, \\ Japan \\ ${ }^{2}$ COSEL CO., LTD, Japan \\ ${ }^{1}$ masashi.ohchi@it-chiba.ac.jp
}

\begin{abstract}
It is necessary to measure and control electric power in the Home Energy Management System (HEMS). Although many smart taps have already been developed for HEMS, they are not used widely in homes because they do not have enough usefulness that deserves their prices. In this study, we develop a tap that has an additional useful functionality; that is, it can cut off a current to prevent whole-house electricity outages. The developed tap is able to measure the waveforms of a voltage and a current, and it can cut off the current more quickly than circuit breakers used in homes. The tap consists of a relay, an electric energy metering IC, and a Wi-Fi module, and can be manufactured at low costs.
\end{abstract}

Keywords: Smart tap, HEMS, Electric power measurement and control

\section{Introduction}

In recent years, the use of photovoltaic power generation, wind power generation, and other natural energies has been promoted, and as a result, power supplies affected by the weather and with significant temporal variation are being connected to the power grid. Thus, attention is being increasingly focused on smart grid [1] and Home Energy Management System (HEMS) technologies to ensure a stable supply of electric power. With the use of smart grids, the introduction of smart meters that can replace conventional electricity meters and determine the electric power used in a house on the supply side every 30 minutes is accelerating.

For HEMS, smart taps [2][3][4][5] or smart meters [6][7][8][9] that can identify and control the power consumption of individual residential electronics are used to control energy use. However, compared with smart meters, the use of smart taps has not yet become widespread. Currently, a tradeoff relation exists with smart taps between higher performance and cost, and one factor that is thought to hinder the widespread use of smart taps and prevent users from experiencing their convenience firsthand is the cost burden on them.

We focused our attention on residential electrical outages due to the operation of residential wiring circuit breakers, and more specifically on the overall load connected to a circuit breaker and its operation. With that focus and with an awareness of manufacturing costs, we embarked on the development of a tap that can measure and control electric power in real time for the operation of the wiring circuit breaker to be prevented till the possible extent [10]. Additionally, our goal was to avoid the operation of the circuit breaker using the developed tap to rapidly estimate the overcurrent of a circuit breaker prior to its operation, and then cut off current, primarily to lower-priority devices, for which power cutoff would 
not cause a major impact. As a result, highly important loads, such as those of medical devices and of computers, can be continuously utilized.

In this paper, we examine the development of a tap using a commercially available, relatively inexpensive Peripheral Interface Controller (PIC) microcomputer, as well as its function to avoid the operation of a circuit breaker based on actual measurements. By measuring the operation time of a residential circuit breaker, we confirmed that several seconds are required for a circuit breaker to trip. Thus, for developing this tap, we targeted an electric current cutoff within a second. The tap that we developed uses a Wi-Fi module to connect with a personal computer (hereinafter, abbreviated as PC) via a wireless LAN, and can cutoff electric current within approximately $0.6 \mathrm{~s}$, even when simultaneous multiple taps are used. The details are reported herein.

\section{Tap developed for real-time measurement and control of electric power}

An exterior view of the tap developed in this research is shown in [Figure 1], and a photo of the internal configuration is shown in [Figure 2]. The performance of the tap is listed in [Table 1].

For developing the tap, we utilized an electric-energy-metering integrated circuit (IC) (Analog Device, Model no. ADE7753, commercial price of approximately 500 yen) to measure electric power; a PIC microcomputer for control (Microchip Technology, Model no. dsPIC33FJ32GP202, commercial price of approximately 400 yen), a latching relay for cutoff (Panasonic, Model no. ADJ23005, commercial price of approximately 800 yen), and a Wi-Fi module (Digi International, WiFi (S6 version) commercial price of approximately 3,500 yen) for communicating with a PC. The components used in this tap are all general-purpose parts that can be inexpensively procured. Additionally, data measured by the tap can be transmitted by wireless LAN to a PC, and current can be cutoff on the basis of a command from the PC.

The developed tap has two measurement modes, and those modes can be switched on the basis of the commands from the PC. Mode 1 transmits effective values for voltage and current, crest value, voltage cycle, and power factor to a PC, and is the mode that is ordinarily used because it employs only a small amount of communication data.

In addition to the data of Mode 1, Mode 2 also transmits waveform data for the voltage and current. This mode employs a large amount of communication data and thus is not ordinarily used.

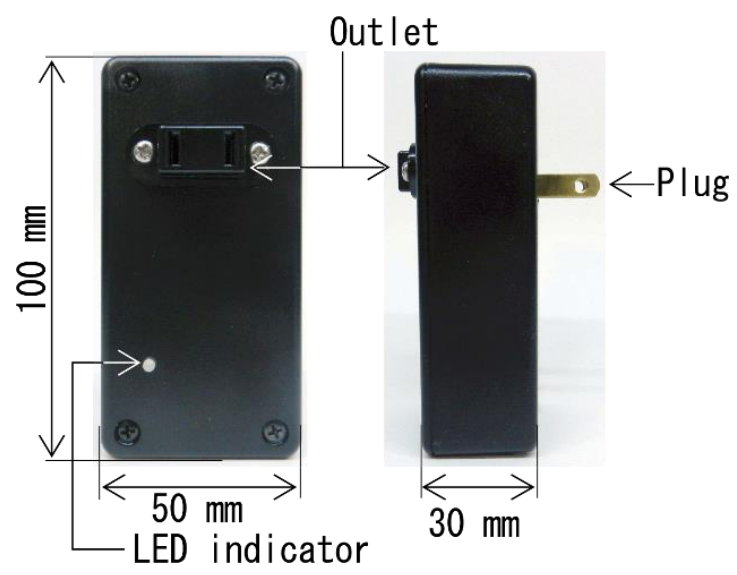

Figure 1. Developed tap 

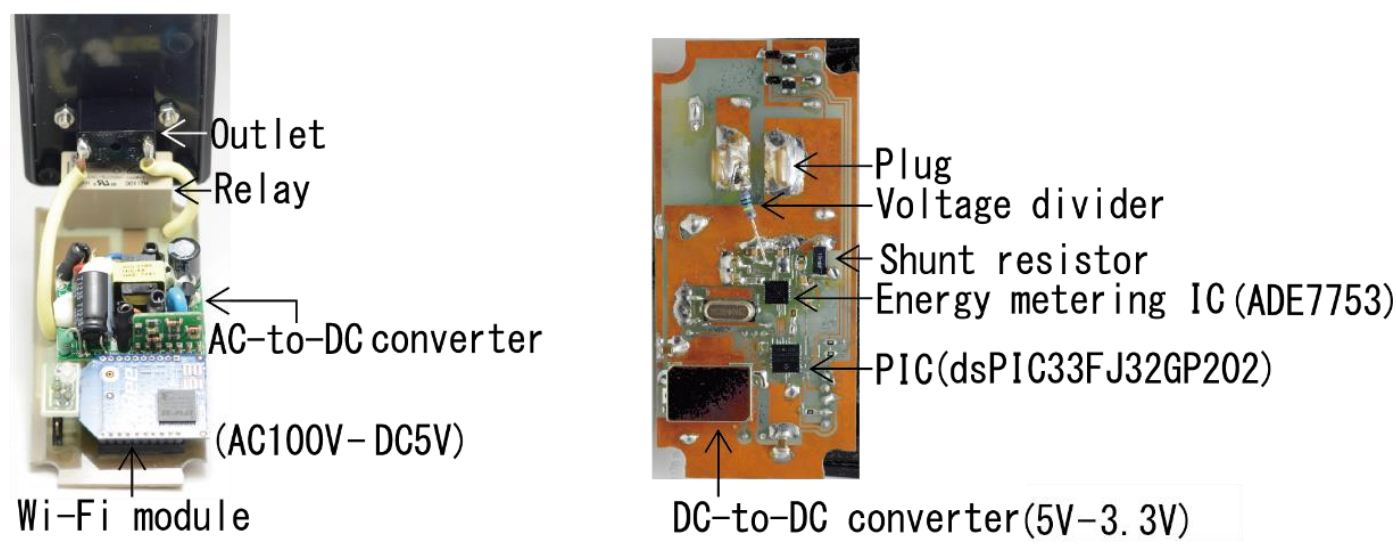

Figure 2. Inside of developed tap

Table 1. Specifications of developed tap

\begin{tabular}{|c|c|c|}
\hline Item & Value & Remarks \\
\hline Rated Voltage & $100 \mathrm{~V}$ & Max $240 \mathrm{~V}$ \\
\hline Rated Current & $15 \mathrm{~A}$ & - \\
\hline A/D Converter (ADC) & Second-order $\Sigma-\Delta$ & ADE7753 \\
\hline ADC Resolution & $24 \mathrm{bit}$ & ADE7753 \\
\hline ADC Sampling frequency & $895 \mathrm{kHz}$ & ADE7753 \\
\hline ADC Bandwidth & $40 \sim 1230 \mathrm{kHz}$ & ADE7753 \\
\hline PIC & $40 \mathrm{MIPS}$ & dsPIC33FJ32GP202 \\
\hline Power Control Device & Relay & Latching Relay \\
\hline Communications Standard & IEE802.11 b/g/n & Wi-Fi \\
\hline AC Power Plugs & $2 \mathrm{P}$ & - \\
\hline AC Power Outlet & $2 \mathrm{P}$ & - \\
\hline Power Consumption & $1 \mathrm{~W}$ & \\
\hline
\end{tabular}

The effective voltage value measured by the developed tap in Mode 1 was compared with the effective voltage value measured using a digital power meter (Yokogawa Meters \& Instruments, Model no. WT210), and both values matched within $\pm 0.01 \%$. Similarly, when the effective current value measured by the developed tap was $0.4 \mathrm{~A}$ or greater and was compared with a measurement obtained using the digital power meter, the two values matched within $\pm 3 \%$. However, when the measurement was less than $0.4 \mathrm{~A}$, the amount of DC offset of the electric-energy-metering IC influenced the measurement, and resulted in a margin of error of $\pm 20 \% \sim 45 \%$. The power-factor measured for the tap was also compared with the measurement from the digital power meter, and the two measurements matched within $\pm 3 \%$.

\section{Real-time measurement of electric power and evaluations}

\subsection{Testing method}


The time between the detection of an overcurrent by the circuit breaker and tripping was measured. Then, the aim was to control this trip time with a tap, and achieve a time that avoids the operation of the circuit breaker. Next, the measurement results of the tap were collected by the PC, and the time between the PC-detected overcurrent and a trip command send by the PC to the tap and the execution of the trip was measured.

On the basis of these two measured durations, we considered whether avoiding the operation of the circuit breaker would be possible.

\subsection{Simulated distribution board}

A simulated distribution board, shown in [Figure 3], was fabricated for testing. The board is configured such that a circuit breaker with a rated current of $20 \mathrm{~A}$ is connected to a commercial power supply, and power is supplied from there to $15,10,5$, and $3 \mathrm{~A}$ circuit breakers. The simulated distribution board is configured with the intention that the developed tap can be attached to each wiring plug connector (hereinafter, "outlet"), and the installation position can be changed on the basis of the testing method.

The measurement environment is shown in Figure 4. The trip time of each circuit breaker installed in the simulated distribution board was measured. When the circuit breakers were used at the rated current, a load of $15 \mathrm{~A}$, which is the maximum current per single load, was connected, resulting in an overcurrent. The current was measured every $0.1 \mathrm{~s}$ using a digital power meter, and the results were recorded with a PC. The duration between the point where current increased to $15 \mathrm{~A}$ and till it became $0 \mathrm{~A}$ was measured; this value was used as the trip time.

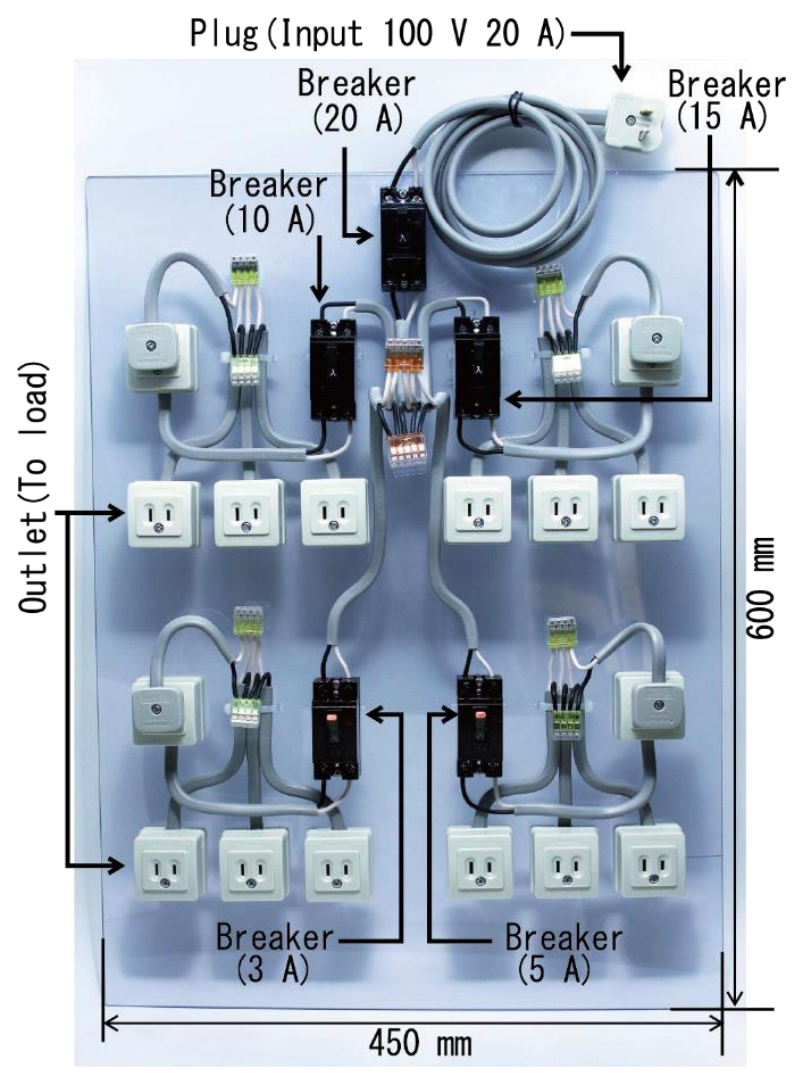

Figure 3. A simulated distribution board 


\subsection{Circuit breaker trip time}

The measurement results for the circuit breaker trip times are listed in [Table 2]. When a total of $25 \mathrm{~A}$ was sent through a circuit breaker, i.e., with a rated current of $10 \mathrm{~A}$ (the current of $10 \mathrm{~A}$ was flowed for $30 \mathrm{~s}$ or longer) and then a current of $15 \mathrm{~A}$, the time required for the circuit breaker tripping averaged $4.0 \mathrm{~s}$ over 10 runs. According to the specifications of the circuit breaker with a rated current of $10 \mathrm{~A}$, the trip time was a minimum of approximately 1.5 seconds. Similarly, when 15 A were sent through a circuit breaker with a rated current of $15 \mathrm{~A}$ and then a total of $30 \mathrm{~A}$, the time required for the circuit breaker to trip averaged $2.2 \mathrm{~s}$. Furthermore, according to the specifications of the circuit breaker with a rated current of $15 \mathrm{~A}$, the trip time averaged $7.0 \mathrm{~s}$.

Table 2. Results of measurement of circuit breaker trip time

\begin{tabular}{|c|c|c|}
\hline $\begin{array}{c}\text { Circuit breaker } \\
\text { rated current } I_{r}[\mathrm{~A}]\end{array}$ & $\begin{array}{c}\text { Measurement average trip } \\
\text { time } t_{a}[\mathrm{~s}]\end{array}$ & $\begin{array}{c}\text { Circuit breaker specification } \\
\text { minimum trip time } t_{s}[\mathrm{~s}]\end{array}$ \\
\hline 10 & 4.0 & 1.5 \\
\hline 15 & 2.2 & 7.0 \\
\hline
\end{tabular}

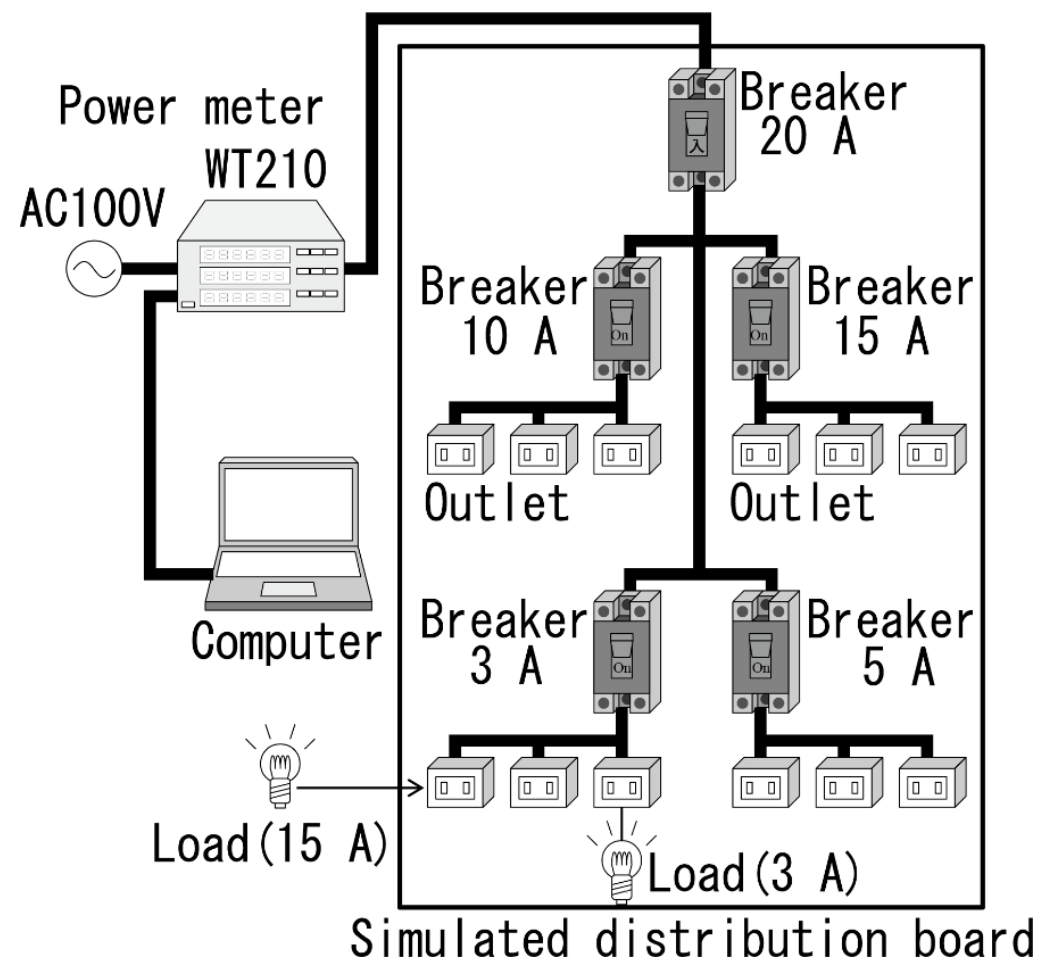

Figure 4. Schematic circuit diagram of estimation board for measuring trip-time of circuit breaker

On the other hand, the 3 and 5 A rated-current circuit breakers with built-in instantaneous tripping devices immediately tripped, making it impossible to obtain measurements. However, it is believed that many general households do not have $3 \mathrm{~A}$ or $5 \mathrm{~A}$ rated-current circuit 
breakers installed for the most part, and therefore, the discussion of this paper focuses on 10 and $15 \mathrm{~A}$ rated current circuit breakers.

\section{Real-time measurement and control testing of electric power}

\subsection{Usage load}

Mode 2 of the tap developed in this research was used for real-time measurement and control testing of individual appliances with different types of electrical power usage, and the operating voltage - current waveforms of the load were measured. The current waveform of an incandescent light bulb (Ohm Electric, Model no. LW100V95W2PA) is shown in [Figure 5]. From this figure, apparently, the power factor is approximately 1 with a linear load.

The current waveform of a fluorescent lamp for ultraviolet light (Sunhayato, Model no. BOX-W9B) is shown in [Figure 6]. In this case, while the load is linear, the power factor is 0.57 , which is the lowest of the loads studied. This is thought to be due to the impact of the fluorescent lamp stabilizer, which uses a choke coil.

The current waveform of an electric vacuum cleaner (Hitachi, Ltd., Model no. CV-PL700) is shown in [Figure 7]. The load is nonlinear, and as is clear from this figure; the peak current is highest at approximately $28 \mathrm{~A}$.

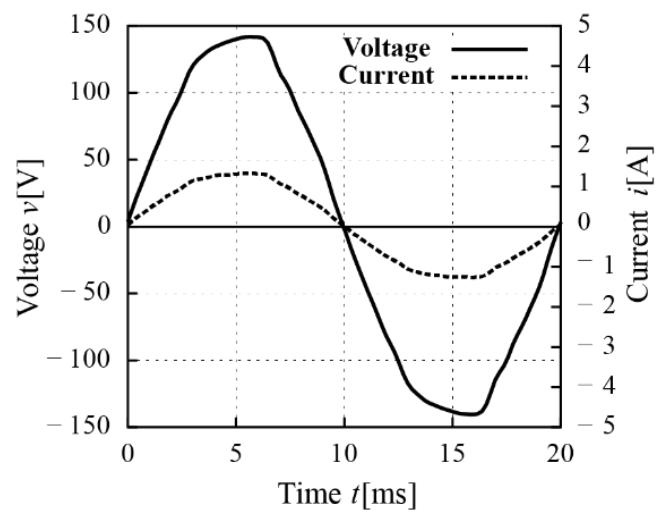

Figure 5. Waveforms of a filament lamp

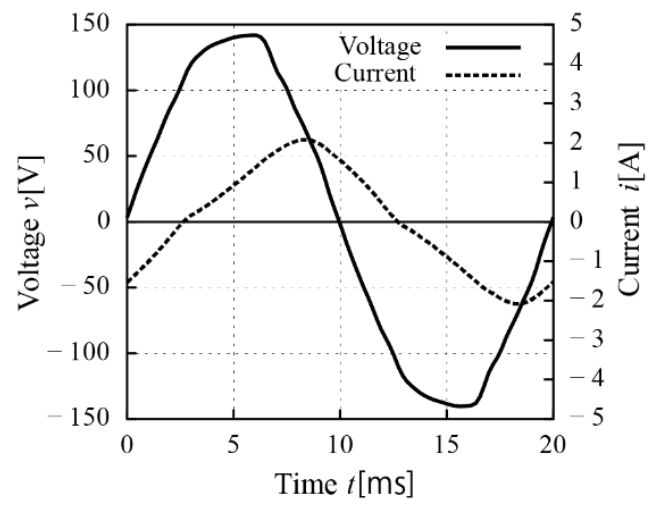

Figure 6. Waveforms of a fluorescent lamp 


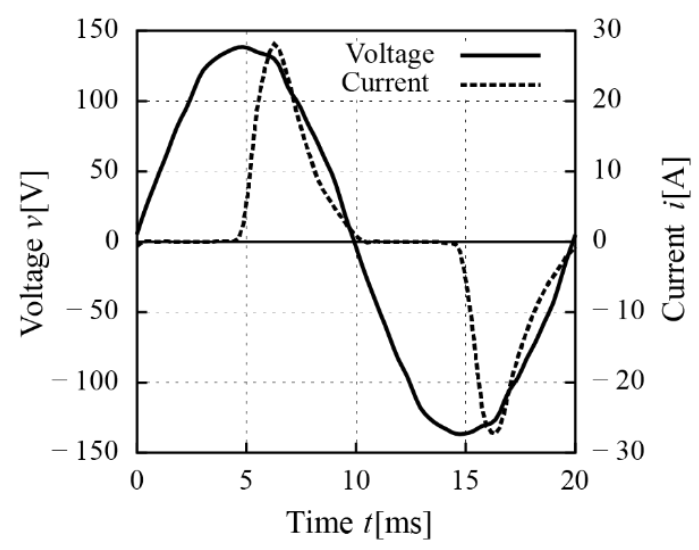

Figure 7. Waveforms of a cleaner

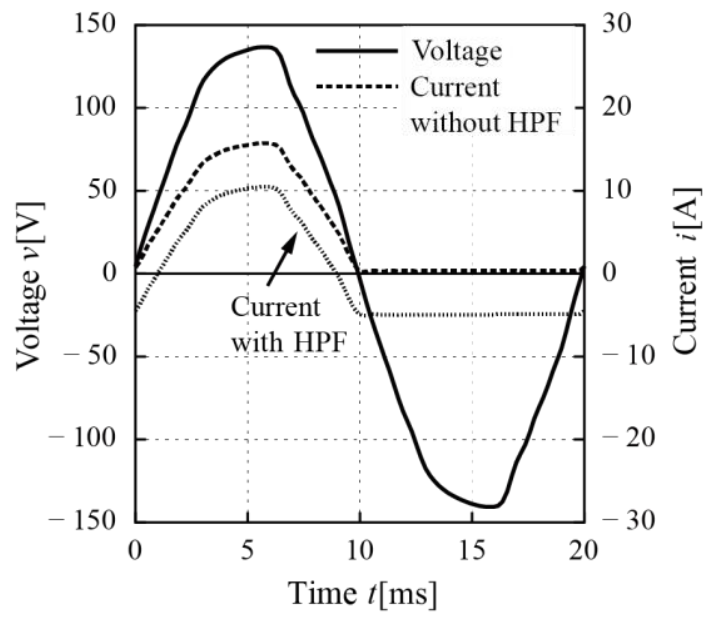

Figure 8. Waveforms of a hair dryer (low setting)

The current waveform of a hair dryer (Koizumi, Model no. KHD-1236), on the low hot-air setting, is shown in Figure 8. When the hair drier is operated, electric current that has been rectified by a diode is supplied to the electric heating wire, and thus, a half-wave rectified current waveform is observed. Here, the electric-energy-metering IC (ADE7753) used by the tap we developed was set to use a built-in High-Pass Filter (HPF) when the initial current is measured.

However, when the current waveform is half-wave, an unintended offset is generated in the current waveform using an HPF. On the other hand, when the setting is such that an HPF is not used, a DC offset component that is applied to the AD converter for measuring electric current cannot be removed. Thus, when the current is very small, a problem occurs: the current-measurement margin of error increases. Therefore, because the current that should be detected by the tap is the maximum current value, we decided to perform testing with a setting that does not use an HPF in this research.

\subsection{Tap trip time}


Using the testing equipment shown in [Figure 9], we transmitted the data measured in Mode 1 of the tap to the PC, estimated the current of the circuit breakers for electric power distribution with the PC, sent a trip command when it was determined that the rated current value was exceeded, and measured the time required for the tap relay to cutoff the electric current. The equipment used in this experiment was the $15 \mathrm{~A}$ rated-current circuit breaker for power distribution shown in [Figure 9].

Using a digital power meter, we measured the current of the 20 A rated-current circuit breaker for power distribution every 0.1 seconds. A load of 10 A or greater (hair drier on the high hot-air setting [high] to achieve a state of no rectification by the diode) was additionally connected to one tap and operated, and we tried to exceed a total load current of $15 \mathrm{~A}$ at this time. Then, we measured the time it took from the point when the current measured by the digital power meter began to increase until the tap cutoff the load on the basis of a command from the PC and the current returned to the original current value. This time was used as the tap trip time.

Next, we increased the number of taps from one to nine by adding one tap at a time and measured the trip time when a load of 10 A or greater was connected to only a single tap. The average trip time (measured ten times for each quantity) and the standard deviation are shown in Figure 10. On average, the trip time was equal to or lesser than $0.6 \mathrm{~s}$, regardless of the increase in the quantity, and the standard deviation averaged $0.12 \mathrm{~s}$. Furthermore, even with an increase in the number of taps, a decrease in the trip time was not confirmed. However, the standard deviation was somewhat large.

\subsection{Measuring the resultant power from individual power}

In this research, the effective current value of the load was measured in Mode 1 of the tap, and the effective current value flowing in the circuit breaker was estimated by summing each of the effective current values.

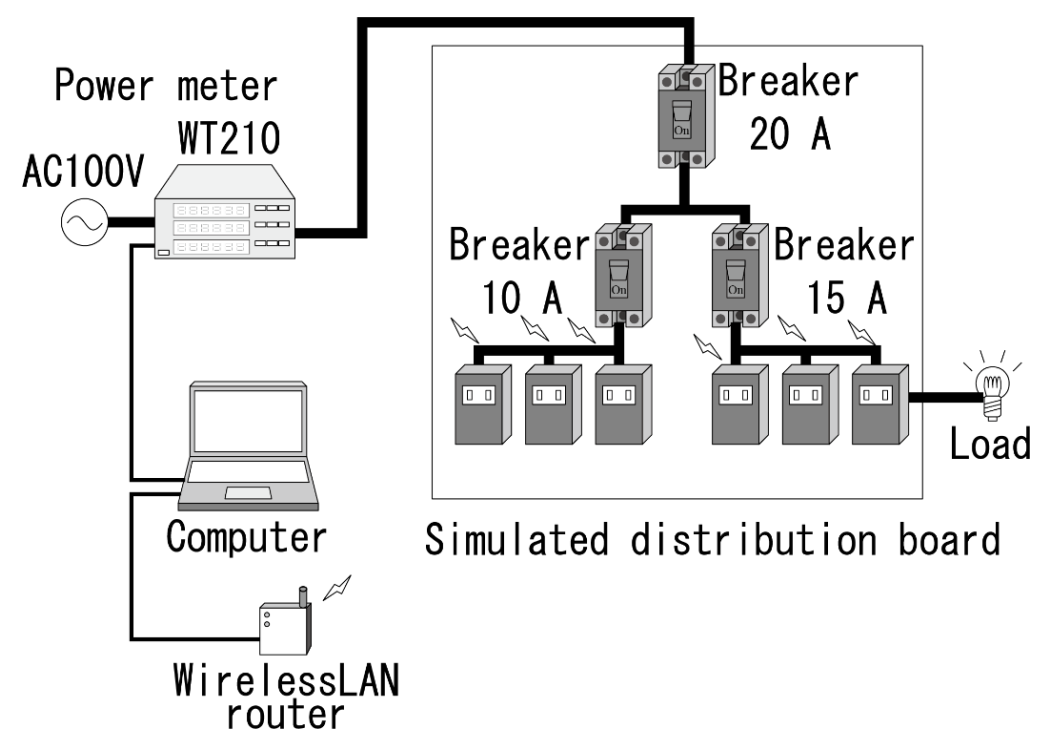

Figure 9. Schematic circuit diagram of estimation board for measuring trip-time of developed tap 


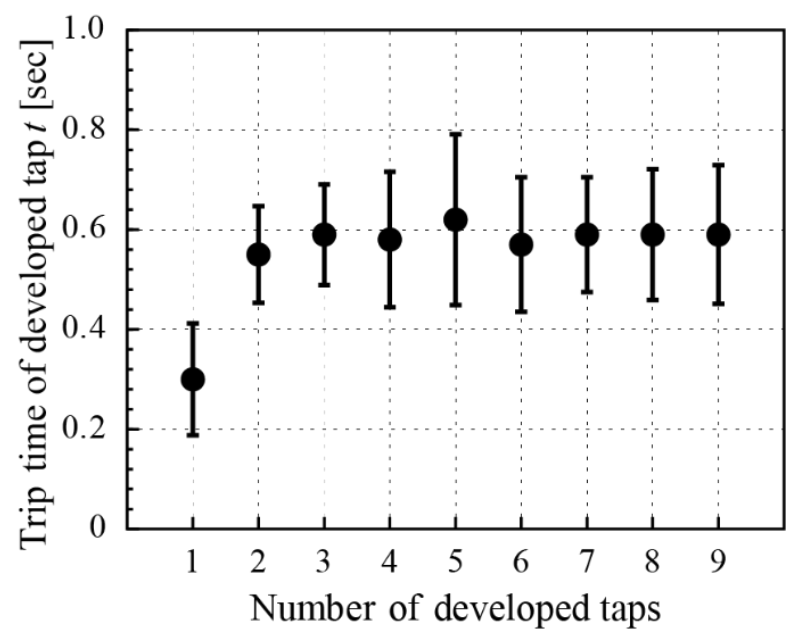

Figure 10. Results of measurement of smart tap trip time

We also developed an application for displaying the estimated effective current value of the circuit breaker for power distribution on a PC. A screen of the PC is shown in [Figure 11]. From this screen, the effective value of the current used by each piece of electronic equipment can be confirmed in real time, and the priority levels that were set when the tap was initially connected can also be confirmed. Those priority levels include "highest," "high," "standard," and "low."

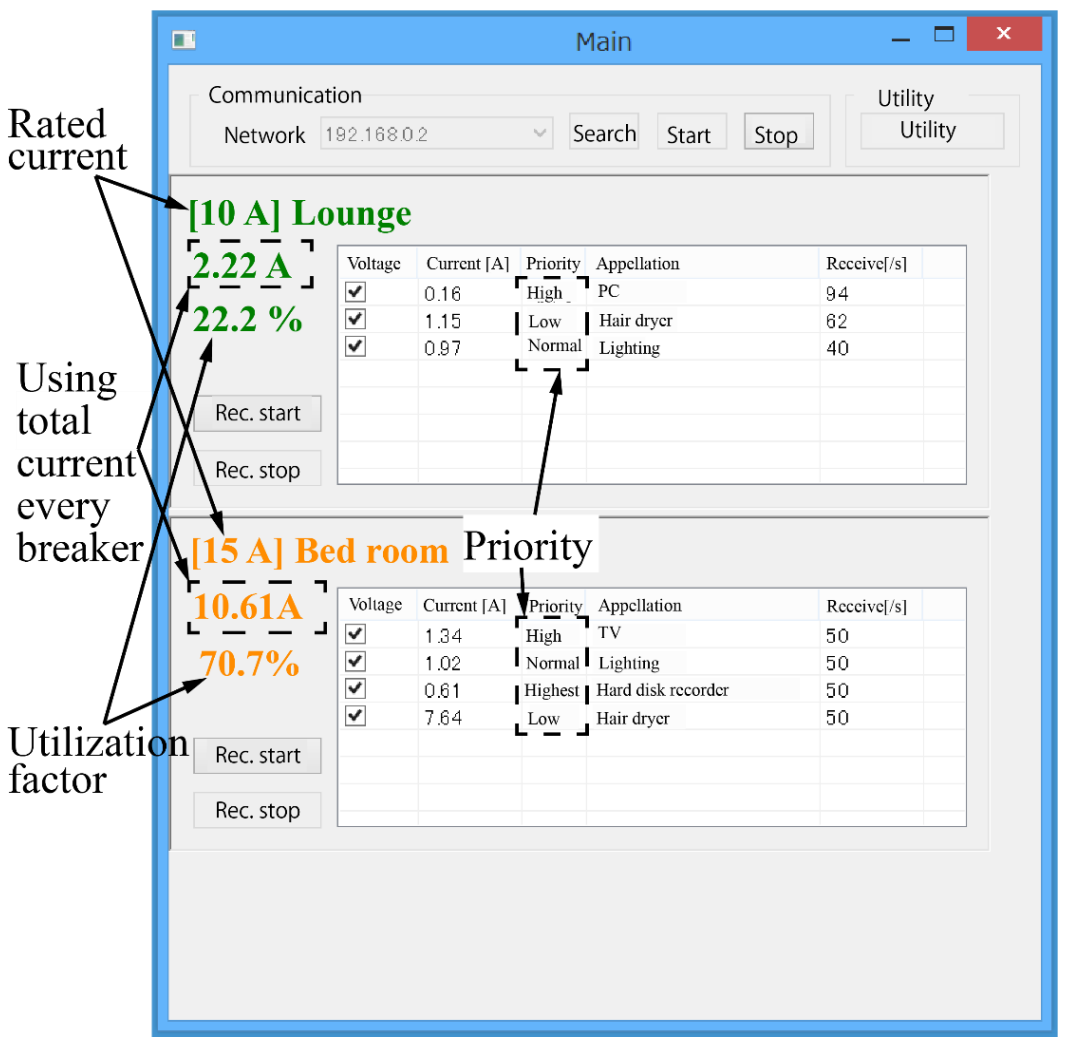

Figure 11. Developed taps management application 
The values that can be measured in Mode 1 of the tap include the effective voltage value, $\mathrm{V}$ (for residential voltage, this value is thought to be constant at $100 \mathrm{~V}$ and thus the measured voltage is a constant value), the effective current value, Ii, and the power factor, $\cos \phi$ (the current value and power factor differ for each tap); using these values, the effective power, $P_{i}$, for each tap can be calculated from Equation (1). Moreover, the reactive power, $Q_{i}$, can also be calculated as shown in Equation (2). Here, the electric energy metering IC used with the tap expresses the power factor as "lagging" or "leading" using signs, and thus, the expression is used to determine the sign of the reactive power.

$$
\begin{aligned}
& P_{i}=V I_{i} \cos \phi_{i} \\
& Q_{i}= \pm \sqrt{\left(V I_{i}\right)^{2}-P_{i}^{2}} \\
& \dot{W}=\sum_{i=0}^{n}\left(P_{i}+Q_{i}\right) \\
& S=V I=\sqrt{P^{2}+Q^{2}} \\
& I=\frac{\sqrt{P^{2}+Q^{2}}}{V}
\end{aligned}
$$

However, the current of each load has different phases, and there are cases of half-waverectified waveforms. Therefore, a margin of error will occur if the effective current values of each load are merely totaled, without consideration of phase, to obtain the effective value of the current flowing to the circuit breaker for power distribution. Hence the overall effective power, $P$, and reactive power, $Q$, as viewed from the circuit breaker for power distribution are determined by summing $P_{i}$ and $Q_{i}$ of each tap, as shown in Equation (3); the apparent power, $S$, is then calculated from Equation (4). Then, a method was used to calculate the effective current value, $I$, of the circuit breaker for power distribution from $S$ using Equation (5).

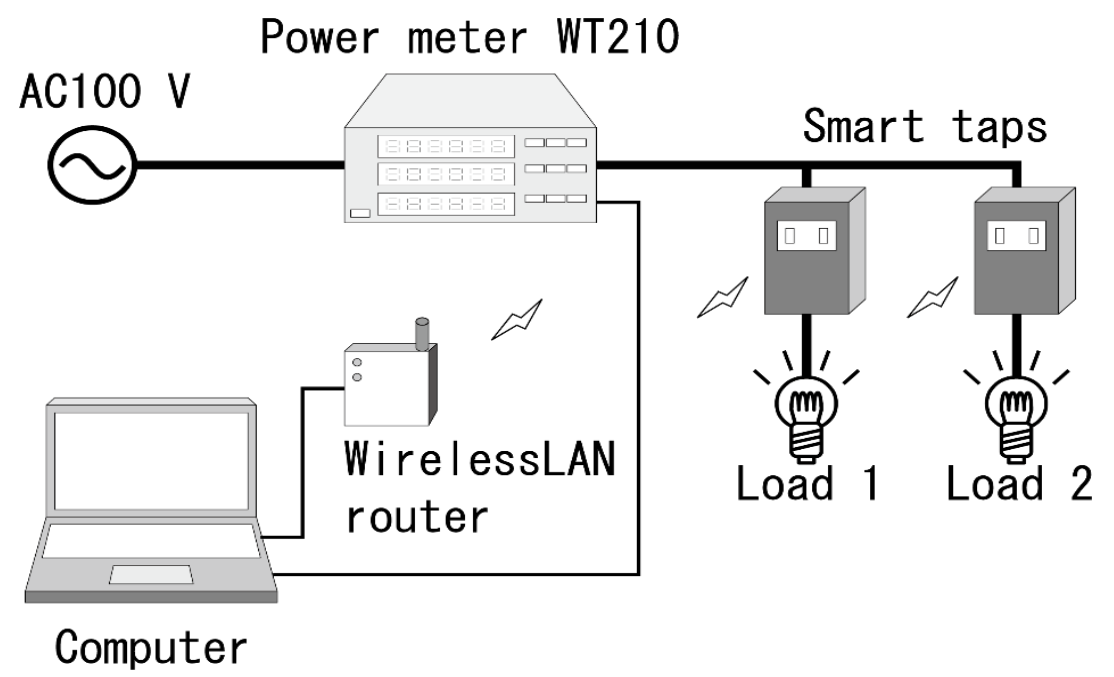

Figure 12. Schematic circuit diagram for measuring current of developed two taps 
As shown in [Figure 12], two loads were connected via smart taps under a single wiring circuit breaker, and the current flowing to the wiring circuit breaker was calculated using the measurements from the taps.

Table 3. Results of measurement of a total of two smart taps current

\begin{tabular}{|c|c|c|c|c|}
\hline \multirow{2}{*}{\multicolumn{2}{|c|}{}} & \multicolumn{3}{|c|}{ average sum current [A] } \\
\cline { 3 - 5 } & Loads & $\begin{array}{c}\text { ST210 bunched } \\
\text { current } I_{W}\end{array}$ & $\begin{array}{c}\text { Smart taps } \\
\text { Vector calculated } \\
\text { current } I_{t p}\end{array}$ & $\begin{array}{c}\text { Directly sum } \\
\text { current } I_{\text {ta }}\end{array}$ \\
\hline Bulb & Bulb & 2.0 & 1.9 & 1.9 \\
\hline Bulb & Fluor lamp & 2.1 & 2.2 & 2.3 \\
\hline Bulb & Cleaner & 13.4 & 13.4 & 13.6 \\
\hline Bulb & Hair drier & 8.7 & 8.6 & 8.8 \\
\hline Fluor lamp & Cleaner & 13.8 & 14.0 & 14.0 \\
\hline Fluor lamp & Hair drier & 8.6 & 9.6 & 20.3 \\
\hline Cleaner & Hair drier & 17.7 & 20.3 & 9.6 \\
\hline
\end{tabular}

As the loads, a linear load (current approaches a sine wave) with a power factor of nearly 1 (white incandescent light bulb), a linear load with a low power factor (fluorescent lamp, power factor $=0.57$ ), and a nonlinear load (current is not a sine wave but rather is a waveform having a half-wave or harmonic wave superimposed) (vacuum cleaner or drier at a low setting) were respectively combined and measured. When the respective loads were combined, the measured values from the taps for the current flowing to the wiring circuit breaker were summed, and were compared by the difference that occurred when the loads thereof were measured simultaneously with a digital power meter. Each combination was measured 20 times. Of course, the power consumption of a single tap is approximately $1 \mathrm{~W}$, and the impact of the power consumed by the two taps when connected to the digital power meter was negligible.

The results for the electric current values obtained by adding the current values measured by each tap and the results for the lump-sum measurements obtained by the digital power meter are shown in [Table 3]. When the total current, $I_{t p}$, flowing to the wiring circuit breaker was calculated using Equation (5) and then compared with the current value, $I_{t a}$, obtained by merely adding the effective current values of the two taps, the two values almost matched. Thus, it is thought that $I_{t a}$ could possibly be used as the estimated current value of the wiring circuit breaker. However, when the drier on a low setting was combined with the fluorescent lamp or the vacuum cleaner, an anomaly occurred such that a difference of approximately $10 \%$ occurred between the current for the wiring circuit breaker, $I_{w}$, measured by the digital power meter and the $I_{t a}$ measured and added by the two taps.

\section{Considerations}

\subsection{Wiring circuit breaker trip time}


We were able to confirm that in some cases, the trip time of the wiring circuit breaker is shorter than noted in the specifications. The shorter trip time is thought to be caused by a flowing overcurrent when the bimetal of the circuit breaker is thoroughly heated, and the bimetal then quickly reached a tripping temperature. Also, for a circuit breaker with a built-in instantaneous tripping device, an inrush current of the load flowing when the power is turned on is thought to cause the instantaneous tripping device to operate, and thus the circuit breaker immediately trips. From the above, we believe that with ordinary wiring circuit breakers that do not have a built-in instantaneous tripping device, one or more seconds are required to detect an overcurrent and then trip.

\subsection{Tap trip time}

The trip time of the tap developed in this research was on average approximately $0.6 \mathrm{~s}$ regardless of the number of taps. Therefore, it is thought that the tap can complete its operation well in advance of the wiring circuit breaker operation. However, while the average trip speed of the tap was a short duration, it became clear that the standard deviation is somewhat large.

\subsection{Calculating the total current from individual currents}

The electric current simultaneously measured and the total value of the electric currents of the taps was generally within \pm 0.1 A. However, depending on the combination of the loads, in certain cases, a difference of $1 \mathrm{~A}$ or greater occurred. The total current of linear loads was effectively calculated, but the results generated a margin of error for nonlinear loads containing a significant harmonic component.

To calculate the total current with good precision in the case of the nonlinear load as well, the Fast Fourier Transform (FFT) is thought as a must to be used for measuring the current values summed for each frequency component, or sampled waveform data is assumed as a must to be transferred to a PC; an algorithm for summing multiple waveforms must then be used on the PC side. However, calculating the FFT requires a significant arithmetic processing capability and a large amount of memory capacity; this requires the introduction of equipment that is more expensive than the PIC microcomputer currently being used.

Moreover, if sampled waveform data are transferred to a PC, the communication load increases, and more time is required to determine an overcurrent state. This leads to the possibility of the inability to trip at high speeds, and new problems arise.

\section{Conclusion}

In this research, we developed a tap that enables inexpensive real-time measurement and control of electric power for HEMS. We measured the operating time of a wiring circuit breaker, and confirmed that approximately 1 second is required for a circuit breaker to tripp. From there, we aimed to develop a tap capable of tripping at a speed higher than 1 second. As a result, we successfully developed a tap that can cutoff electric current according to a priority ranking in approximately 0.6 seconds prior to the operation of a wiring circuit breaker. This is achieved by connecting respective taps between numerous loads connected to a single wiring circuit breaker, and then estimating the overcurrent of the wiring circuit breaker from the electric current measured by the taps.

We also verified that even when a total of nine taps are used, the trip time remains nearly constant. However, when a nonlinear device was connected, in some cases, the total of the 
respective electric current measurement values measured by the two taps exceeded the actual measured current of the circuit breaker for power distribution.

In other words, it became clear that an accurate current value cannot be estimated by merely totaling the effective values for the electric current; this results in the problem of current being cut to low-priority devices by the taps even when the rated current of the wiring circuit breaker has not been reached. However, from the test results this time, because the rated current of the wiring circuit breaker was not exceeded, at the current point in time, we feel that this will not be a major problem.

An issue for the future is to accurately estimate the electric current of a wiring circuit breaker even when load devices having a unique current waveform are connected.

\section{References}

[1] T. Gouda and T. Morozumi, "Smart Grid Textbook," Impress Japan, (2011)

[2] T. Kato and T. Matsuyama, "Electrical Power Visualization System based on Smart Tap Network," IPSJ SIG Technical Report, vol.2011, no.6, pp.1-6, (2011)

[3] M. Tsukamoto and T. Kato, "i-Energy: electric power management by information technologies: developing common specifications of smart-taps," IPSJ, vol.51, no.8, pp.934-942, (2010)

[4] N. Morimoto, M. Tanaka, T. Akehi, M. Yoshida, H. Yoshimizu, M. Takiyamada, and Y. Kamimura, "A smart tap for intelligent and active power control,” IPSJ, Kansai2011084, no.101, (2011)

[5] Y. TAKABAYASHI, H. FUSE, Y. NOMOTO, M. ISSHIKI, and K. ABE, "Evaluation of human behavior estimation of smart tap using self-diagnostics," 2019 IEEE International Conference on Consumer Electronics (ICCE), Las Vegas, NV, USA, pp.1-6, (2019) DOI: 10.1109/ICCE.2019.8661977

[6] D. Xiong, Y. Chen, X. Chen, M. Yang, and X. Liu, "Design of power failure event reporting system based on nb-iot smart meter," 2018 International Conference on Power System Technology (POWERCON), Guangzhou, pp.1770-1774, (2018) DOI: 10.1109/POWERCON.2018.8601747

[7] F. Yang, X. Hou, G. Pan, Q. Luo, and C. Huang, "Research on reliability modeling and evaluation method of smart meter," 2018 IEEE International Conference on Automation, Electronics and Electrical Engineering (AUTEEE), Shenyang, China, pp.244-248, (2018) DOI: 10.1109/AUTEEE.2018.8720801

[8] S. A. Tijani and K. Butler-Purry, "Investigation of approaches for incorporating smart meter data in load and pv models in medium voltage power system studies," 2019 IEEE Texas Power and Energy Conference (TPEC), College Station, TX, USA, pp.1-5, (2019) DOI: 10.1109/TPEC.2019.8662172

[9] W. Shuang, Z. Ting, W. Xiaodong, D. Xiaomeng , and Z. Jia, "Research on the analysis method of the total power value change in the smart meter," 2019 IEEE 3rd Information Technology, Networking, Electronic and Automation Control Conference (ITNEC), Chengdu, China, pp.2282-2285, (2019) DOI: 10.1109/ITNEC.2019.8729379

[10] T. Tanaka, and M. Ohchi, "Development of real-time electric power measurement taps," Annual Meeting Record IEE. Japan, vol.1, no.1-093, pp.118, (2013)

\section{Authors}

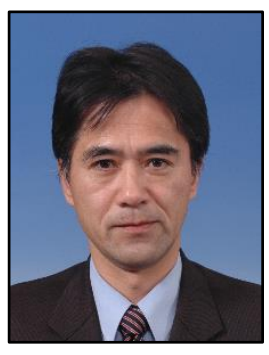

\section{Masashi Ohchi}

He received B.E and M.E degrees in 1987 and 1989 respectively from Nagasaki University, Nagasaki, Japan. He joined Nihon Telephone Telegraph Co. in 1989. He was adopted as a research associate at Saga University, Saga, Japan. He received the D.E degree from Kyushu University, Japan in 2000. He was an associate professor of the Department of Electrical and Electronic Engineering at Saga University since 2000. He has moved to Chiba Institute of 
Technology, Chiba, Japan as a professor in 2008. He is a professor of the Department of Electrica1, Electronics and Computer Engineering at Chiba Institute of Technology. He is a member of The Institute of Electrical Engineers Japan, The Institute of Electronics, Information and Communication Engineers Japan. His research fields are the electrostatic field simulation technology, the ultra-precise instrumentation on the small capacitances.

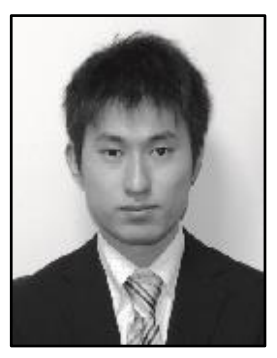

\section{Tomonobu Tanaka}

He received B.E and M.E degrees in 2012 and 2014, respectively from the Chiba Institute of Technology. He is with COSEL CO., LTD. since April 2014. 